\title{
Advances in Microwave-Assisted Freeze Substitution
}

\author{
David W. Dorward, Jennifer Raae-Nielsen, Bryan T. Hansen, Vinod Nair, and Elizabeth R. Fischer
}

Electron Microscopy Unit, Research Technologies Branch, NIAID, NIH, Rocky Mountain

Laboratories, 903 South Fourth St., Hamilton, MT USA

Cryofixation followed by freeze substitution is considered an optimal approach at stabilizing and contrasting hydrated samples for microscopy that are too thick for direct cryoimaging [1]. Because the first step in freeze substitution involves replacement of ice within the sample with a mixture of fixative(s) in solvent at temperatures below -70 degrees $\mathrm{C}$, processing protocols often extend into several days allowing for complete diffusion of reagents into the frozen sample, and for fixation reactions to occur. This process has been shortened considerably using a pre-cooled sample cryoblock placed on a labortory shaker [2]. However, because that system lacks temperature regulation it is unclear whether substitution and fixation occur at equivalent temperatures throughout the frozen sample. Furthermore, cryovials containing fixatives and solvent that are sealed at liquid nitrogen temperature and warmed to room temperature pressurize during the process creating a risk of rupture.

In order to achieve rapid and reproducible freeze substitution for light and electron mcroscopy, our laboratory has developed a system for regulating cryosample temperature within a standard commercial microwave processor. An initial system for microwave-assisted freeze substitution (MWFS) was introduced in 2011 [3]. That prototype proved effective at freeze substitution of cryo samples up to 3-4 $\mathrm{mm}$ in diameter within about 90 minutes after sample introduction. This study introduces a modified version of the device that increases the stability and rates of cooling and heating (Figure 1), and minimizes the quantity of liquid nitrogen used during processing.

Figure 1 shows the system mounted in a model 3751 microwave processor (Ted Pella, Inc., Redding, CA) (Figures 1A-B). The cryo-thermal system is manufactured by Total Temp Technologies, San Diego, CA, and can be installed in most, perhaps all commonly available variable-wattage processing ovens. Temperature is regulated by a programmable controller that balances liquid nitrogen flow rate and in-block heating elements. The controller accepts manual, programmed, and triggered inputs. It also records and tabulates operating parameters such as program steps, time, and temperature. Dry-gas purge provides condensation control (not shown). A temperature response graph from a recent cell layer processing run is shown in Figure 1C. Because sample vessels are exposed to ambient temperature in the microwave chamber, a differential occurs between sample well and platform temperature. The differential varies by: platform versus ambient temperature, the nature, number, and volume of sample(s), the microwave wattage and duty cycle, and the volume of water load in the chamber. Optimal and reproducible parameters are determined quickly using mock samples. Figure 1D shows a section of cryofixed Bacillus subtilis, processed and ready for embedding in about 70 minutes.

Using this system samples placed in the unit are typically transferred into embedding medium after 7075 min. Low temperature infiltration with acrylic embedding resins such as Lowicryl HM20® can be completed by MWFS in under an additional hour. Multiple processing runs can be performed daily. By tightly regulating time and temperature, parameters can be optimized for a wide variety of cryo samples and fixative/solvent formulations for both light and electron microscopy, with excellent preservation of structure and antigenic activity for immunogold and immune histochemical labelling. 
References:

[1] RD Leapman, Cur. Opin. Neurobiol. 14 (2004) p. 591.

[2] KL McDonald, RI Webb, J. Microsc. 243 (2011) p. 227

[3] D Dorward, et al, Microsc. and Microanal. Proc. (2011) p. LB021CD.

[4] The authors acknowledge this research was supported by the Intramural Research Program of the NIH, NIAID, which has a patent pending for this technology.
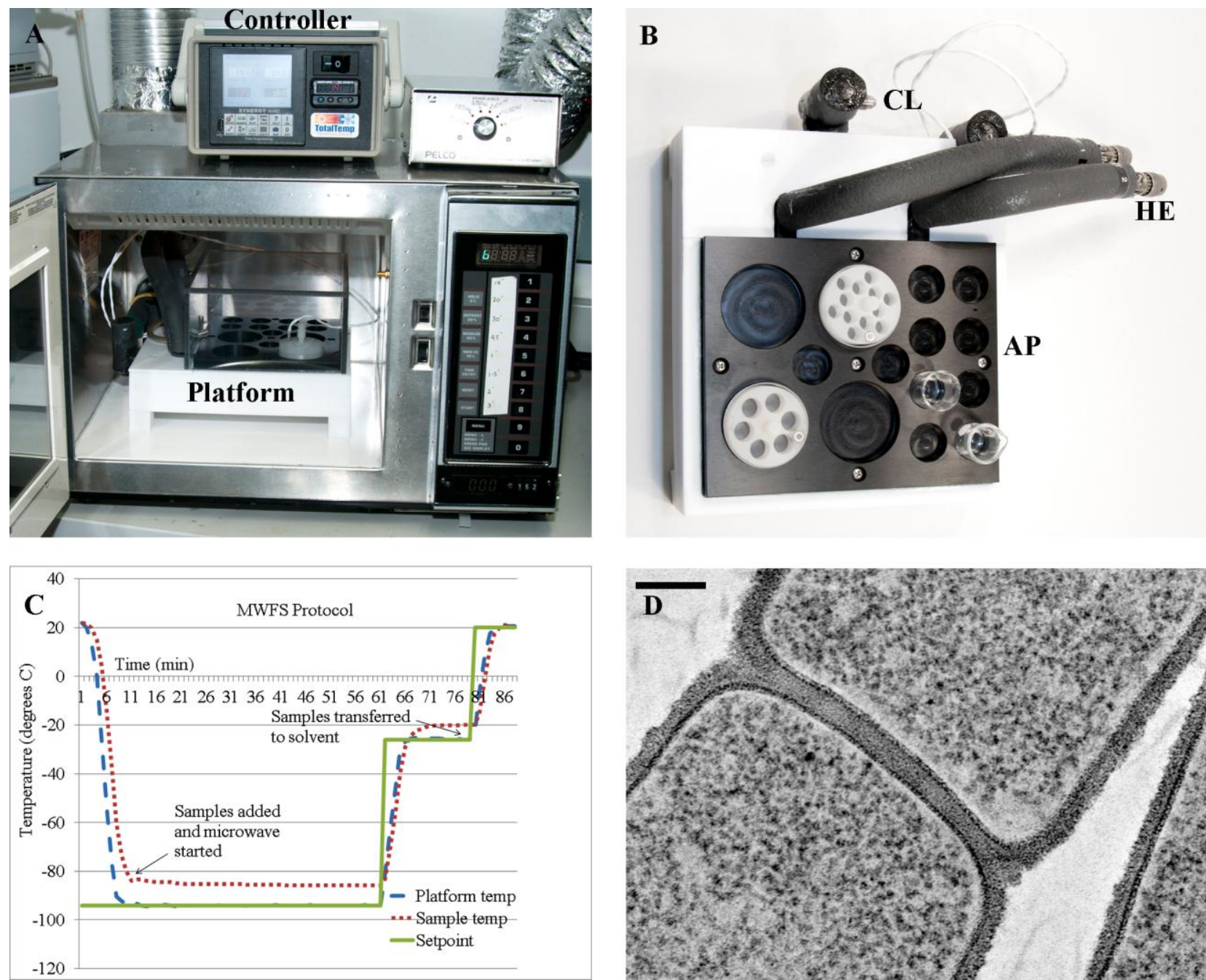

Figure 1. Cryo-thermal platform for MWFS. Figure 1 depicts the MWFS system (A), the cryo-thermal platform (B), a temperature response graph from a processing run (C), and a section from a highpressure-frozen sample of $B$. subtilis prepared by MWFS in acetone containing $1 \% \mathrm{OsO}_{4}$ and $0.1 \%$ uranyl acetate (D). The system consists of a programmable controller that regulates cooling and heating between -100 and 100 degrees $\mathrm{C}$, the cryo-thermal platform, temperature probes (white wires), a 30L pressurized liquid nitrogen dewar, and an insulted hose (not shown). The platform is a removable Teflon ${ }^{\circledR}$ block with cryogen lines (CL) and electric heating elements (HE). Samples are placed within recesses tooled in an anodized aluminum adapter plate (AP), which is configured to user specifications. Panel $\mathrm{C}$ shows an Excel ${ }^{\circledR}$ graph of an MWFS run for 50 min below $-80^{\circ}, 10$ min at $-20^{\circ}$, with removal at $20^{\circ}$, using microwave irradiation of $80 \mathrm{~W}, 70 \%$ duty cycle, and $3 \times 1 \mathrm{~L}$ water load. After embedding and sectioning, B. subtilis processed by MWFS is well preserved and contrasted. Bar: $100 \mathrm{~nm}$. 